\title{
Childhood obstructive sleep apnoea: to treat or not to treat, that is the question
}

\section{Carole L Marcus}

Childhood obstructive sleep apnoea (OSA) is relatively common, occurring in at least $2 \%$ of children ${ }^{1}$ with other studies estimating a much higher prevalence. ${ }^{2}$ Part of the reason for the controversy regarding the prevalence of OSA is the lack of a standardised definition. In children with OSA, clinical symptoms during wakefulness tend to be vague and non-specific (eg, behavioural issues) and are often attributed to other problems; excessive daytime sleepiness is relatively uncommon. Relying on diagnostic polysomnography is also problematic. An apnoea-hypopnoea index (AHI) $\geqslant 1.5 / \mathrm{h}$ is considered statistically abnormal..$^{3-5}$ However, this does not mean that every child with an AHI $\geqslant 1.5 / \mathrm{h}$ will benefit from treatment. There is a paucity of data on the clinical outcomes of children with OSA, and virtually no data on the clinical correlates of polysomnographic abnormalities. The usual treatment for OSA in young children is adenotonsillectomy. Should children with mild OSA be subjected to this surgery, with all of its potential attendant complications? To resolve this controversy, we need the answer to two questions:

- What is the clinical outcome of mild OSA?

- What is the natural history of mild OSA if left untreated?

In this issue of Thorax, Li et al describe the natural history of mild OSA (AHI 1$5 / \mathrm{h}$ ) in 6-13-year-old children over the course of 2 years (see page 27). The authors found that, as a whole, the children had a mild improvement in AHI. However, $29 \%$ of the children had a worsening of OSA. In some cases the OSA progressed into the moderate or severe range. The children who were most likely to have worsening of OSA were younger, more likely to be male and more likely to have large tonsils. The worsening

Correspondence to: Dr C L Marcus, Children's Hospital of Philadelphia, Pulmonary Division, 5th Floor Wood, 34th and Civic Center Blvd, Philadelphia, PA 19104, USA; marcus@email.chop.edu in the younger children may have been due to the increased propensity of the lymphoid tissue to proliferate in this age range. The authors also noted that children with a larger increase in waist circumference were more likely to have worsening of their OSA. However, this finding should be interpreted with caution as the authors used the increase in absolute waist circumference rather than the $z$-score in their final multivariate model, and waist circumference will obviously increase with growth. Of note, the body mass index z-score was not predictive of worsening OSA.

This study is important for several reasons. No other study has evaluated the natural history of OSA in children. It would be expected that OSA would change in children as they grow due to changes in airway calibre, potential decreases in upper airway lymphoid tissue and the secretion of sex hormones during puberty. Thus, it is possible that OSA could either improve or worsen if left untreated.

The natural history of childhood OSA is a difficult issue to study as, ideally, large cohorts of untreated children would need to be evaluated for many years. Such a study would be ethically challengingly and financially burdensome. Retrospective studies are impractical due to the changing epidemiology of adenotonsillectomy surgery; back in the 1950s adenotonsillectomy was a rite of passage for most children, whereas currently OSA is the primary indication for surgery. Thus, the current study of children with mild OSA, although small and limited in duration, is an important first step in determining the natural history of mild OSA and provides valuable and clinically relevant information.

How can this study guide us in the management of children with mild OSA? It should be noted that, while $29 \%$ of children worsened over time, in general the AHI actually improved significantly for the group as a whole. This suggests that watchful waiting may be an acceptable management plan in children with mild OSA who meet certain criteria: older age, small tonsils and perhaps not obese.
However, this begs a bigger question: What exactly constitutes mild OSA in children? Statistically, an AHI $\geqslant 1.5 / \mathrm{h}$ is abnormal in children. ${ }^{3-5}$ In contrast, an AHI $\geqslant 5 / h$ is considered abnormal in adults. However, just because an AHI is statistically abnormal, it is not necessarily clinically significant. While few well-controlled studies are available, some data suggest that even mild OSA is associated with neurobehavioural abnormalities that improve following surgery. ${ }^{8}$ Similarly, evidence suggests that even very mild OSA can affect blood pressure regulation, ${ }^{10}$ and even mild nocturnal desaturation can impair growth in children with bronchopulmonary dysplasia. ${ }^{11}$ However, all of the studies evaluating morbidity of mild OSA have been limited by small cohorts, lack of socioeconomically matched controls, lack of randomisation or blinding, or finding differences between groups that nevertheless remain in the normal range. Currently, a large National Institutes of Health randomised doubleblind clinical trial (the Childhood Adenotonsillectomy or CHAT study) is under way which should help determine the outcomes of mild OSA. The CHAT study is evaluating children with an AHI as low as $2 / \mathrm{h}$. If this study shows that even mild OSA is associated with morbidity, then even children at low risk for worsening should undergo treatment of OSA.

Some limitations of this study should be noted. Most importantly, the study only evaluated children who were not treated surgically for OSA. We are not told how many children were treated surgically, or how children and families from the parent study were counselled following the initial polysomnography. It is possible that children with worsening symptoms underwent surgery, which would suggest that the rate of worsening of OSA may be much higher than onethird of patients.

Another caveat is that this study was limited to a small homogeneous group of relatively lean Asian children, ranging in age from school age to early adolescence. It is not clear that these data can be extrapolated to other populations, particularly the much more obese population in Western countries. It would be interesting to study younger children and adolescents separately. The peak age of OSA is in the preschool population, and these children may well have a higher rate of worsening due to increasing adenotonsillar hypertrophy. Conversely, adolescents may also be at increased risk of worsening of OSA due to sex hormone secretion. 
An important next step would be to evaluate the natural history of children who successfully underwent surgical treatment of OSA. Many studies suggest that a significant number of children have incomplete resolution of OSA after surgery, but we do not know how many children worsen over time.

In summary, this is an important first study but much needs to be done before we can decide whether watchful waiting is a valid long-term management plan for children with mild OSA.

Competing interests: None.

Thorax 2010;65:4-5. doi:10.1136/thx.2009.123141

\section{REFERENCES}

1. Redline S, Tishler PV, Schluchter M, et al. Risk factors for sleep-disordered breathing in children Associations with obesity, race, and respiratory problems. Am J Respir Crit Care Med 1999;159:1527-32

2. Lumeng JC, Chervin RD. Epidemiology of pediatric obstructive sleep apnea. Proc Am Thorac Soc 2008;5:242-52.

3. Uliel S, Tauman R, Greenfeld M, et al. Normal polysomnographic respiratory values in children and adolescents. Chest 2004;125:872-8.

4. Traeger N, Schultz B, Pollock AN, et al. Polysomnographic values in children 2-9 years old: additional data and review of the literature. Pediatr Pulmonol 2005; 40:22-30.

5. Witmans MB, Keens TG, Davidson Ward SL, et al. Obstructive hypopneas in children and adolescents: normal values. Am J Respir Crit Care Med 2003;168:1540.
6. Li AM, Au CT, Ng SK, et al. Natural history and predictors for progression of mild childhood obstructive sleep apnoea. Thorax 2009;65:27-31.

7. Erickson BK, Larson DR, St Sauver JL, et al. Changes in incidence and indications of tonsillectomy and adenotonsillectomy, 1970-2005. Otolaryngol Head Neck Surg 2009;140:894-901.

8. Chervin RD, Ruzicka DL, Giordani BJ, et al. Sleepdisordered breathing, behavior, and cognition in children before and after adenotonsillectomy. Pediatrics 2006;117:e769-78.

9. O'Brien LM, Mervis CB, Holbrook CR, et al. Neurobehavioral implications of habitual snoring in children. Pediatrics 2004;114:44-9.

10. Amin RS, Carroll JL, Jeffries JL, et al. Twenty-four-hour ambulatory blood pressure in children with sleep-disordered breathing. Am J Respir Crit Care Med 2004;169:950-6.

11. Moyer-Mileur LJ, Nielson DW, Pfeffer KD, et al. Eliminating sleep-associated hypoxemia improves growth in infants with bronchopulmonary dysplasia. Pediatrics 1996:98:779-83.

\section{Seldinger chest drain insertion: simpler but not necessarily safer}

\section{Nick A Maskell, ${ }^{1}$ Andrew Medford, ${ }^{1}$ Fergus V Gleeson ${ }^{2}$}

A symptomatic pleural effusion is a common cause of presentation to medical admission units across the UK. Traditionally, large-bore Argyle-type drains were inserted but, over the past decade, there has been a move to inserting small-bore 10-12 French gauge drains using the Seldinger technique. ${ }^{1}$ In a recently published report, over 20000 units of one Seldinger-type drain were sold in the UK in 2004. ${ }^{2}$ The reasons for this include a perceived reduction in patient discomfort and invasiveness, and the apparent ease and speed of insertion of the smaller drains. This change in practice has occurred alongside new methods of training junior doctors, with Modernising Medical Careers and the European Working Time Directive leading to a reduction in their total work hours and a move to shift work patterns. This has inevitably led to reduced trainee experience on the "shop floor".

Surveys on chest drain insertion have shown that, even among experienced respiratory physicians and thoracic surgeons, overpenetration of the trocar and visceral injuries using Argyle-type chest drains occur. ${ }^{4}$ This led to suggestions to

\footnotetext{
${ }^{1}$ North Bristol Lung Centre, Southmead Hospital, Bristol, UK; ${ }^{2}$ Department of Radiology, Churchill Hospital, Oxford, UK

Correspondence to: Dr N A Maskell, North Bristol Lung Centre, Southmead Hospital, Bristol BS10 5NB, UK; nick. maskell@bristol.ac.uk
}

improve safety and the removal of trocars from some Argyle drain packs.

It has been assumed that the recent change in chest drain insertion to the use of smaller bore chest drains inserted using the Seldinger technique is safer, but there is at present no evidence to support this assumption. Unfortunately, they may cause the same array of problems in inexperienced hands and may potentially expose the patient to additional risks from the use of the sharp long dilator in the small-bore catheter packs and, as the blunt dissection technique is not used, the intercostal artery may be more vulnerable using this approach. Complications of small-bore chest tubes include:

- Puncture of the intercostal artery.

- Over-introduction of the dilator into the chest cavity causing organ perforation.

- Hospital-acquired pleural infection using a non-aseptic technique.

- Inadequate "stay" suture allowing the chest tube to fall out.

- Tube blockage, which may be more common than with larger bore Argyle drains.

The recent National Patient Safety Agency (NPSA) alert reported 12 deaths and 15 cases of serious harm related to chest drain insertion between January 2005 and March 2008. Common factors related to the incidents included the lack of experience of the operator, an inadequate level of supervision, failure to follow the manufacturer's instructions, choice of a suboptimal insertion site and poor patient positioning, suboptimal imaging and a lack of familiarity with published guidelines ${ }^{5}$ on chest drain insertion. Deaths were secondary to puncture of the heart, lungs and liver (fig 1). Severe harm occurred due to drain insertion on the wrong side, damage to vessels, the trachea and the liver. Moderate harm in most cases related to poor management after the drain had been inserted. The Medicines and Healthcare Regulatory Authority has had nine adverse incidents reported since 2003, all but one related to the use of the Seldinger technique for chest drain insertion. These reported incidents are likely to be a significant underestimate of the actual number that have occurred across the UK during this period. An abstract presented at the 2008 British Thoracic Society winter meeting reported $65 \%$ of trusts had encountered

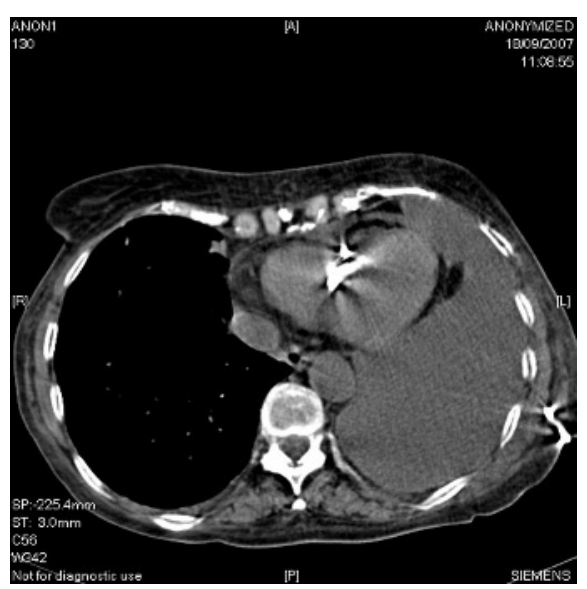

Figure 1 Small-bore chest drain misplaced in left ventricle. 